\title{
Monitoring Bands and Monitoring Rules: how currency intervention can change market composition*
}

\author{
Luisa Corrado $^{\dagger} \quad$ Marcus Miller ${ }^{\ddagger} \quad$ Lei Zhang§ $^{\S}$
}

25 August 2006

\begin{abstract}
In this paper we show how trading rules can generate excess volatility in the exchange rate through repeated entry and exit of currency "bears" and "bulls". This is something of a caricature: but it allows us to show that official action can have self-fulfilling effects as market composition shifts in ways that support official stabilization. Intervention if and when the rate moves outside what Williamson has labelled 'monitoring bands' can reduce market volatility as the effect of the policy is to select endogenously traders from the market whose expectations match official intervention.
\end{abstract}

JEL: D52, F31, G12.

Key-Words: Monitoring Rules, Monitoring Band, Bear and Bull Traders, Excess Volatility, Central Bank Volatility.

${ }^{*}$ Luisa Corrado gratefully acknowledges the Marie-Curie Intra European Fellowship 514913. Marcus Miller and Lei Zhang gratefully acknowledge the financial support of ESRC Grants RES-051-270125 and RES-156-25-0032 respectively.

${ }^{\dagger}$ Faculty of Economics, University of Cambridge and University of Rome, Tor Vergata.

${ }^{\ddagger}$ Department of Economics, University of Warwick and CEPR.

$\S^{\S}$ Department of Economics, University of Warwick. 


\section{Introduction}

When the euro was created in 1999 some economists ${ }^{1}$ predicted that it would rise strongly from its launch value of $\$ 1.18$ as it challenged the dollar's position as the world's key reserve currency. Perhaps because Asian countries lost faith in the IMF and turned to the dollar as a substitute, the euro bulls were disappointed; and the new currency steadily lost value, Figure 1. Finally, when the euro fell to 83 cents in September 2000, coordinated Central Bank intervention was implemented to stop the slide. A famously incautious remark by the then head of $\mathrm{ECB}^{2}$ led to further weakness (with the euro falling to its historic minimum a month later); but the euro stabilized around 90 cents. From early 2002, the currency experienced a reversal that was to take it well above the launch price, reaching a peak of $\$ 1.36$ in the end of 2004. Currently, with the euro standing close to $\$ 1.30$, there is still talk of further dollar devaluation to help reduce the US deficit.

European Central B ank (c)1 999-2006

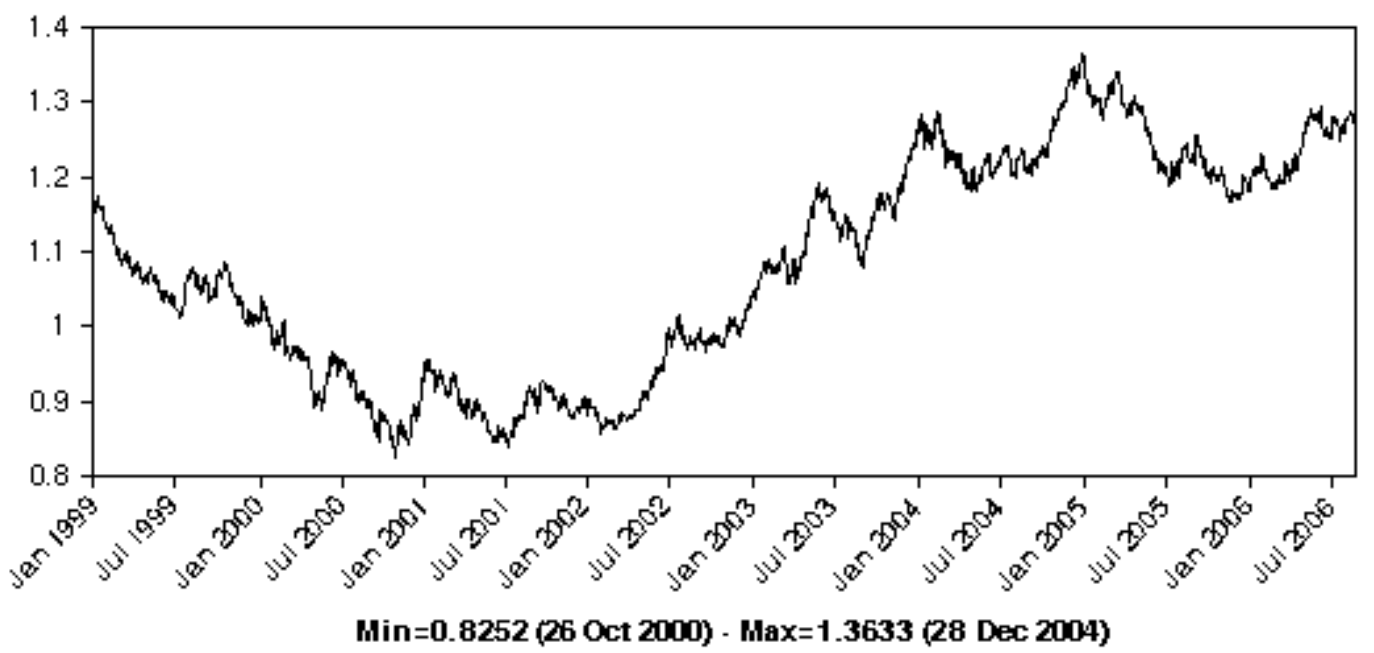

Figure 1: The value of the Euro since its launch in Jan 1999. Source: ECB

The substantial - and surprising - gyrations in the euro/dollar rate have led to calls for the publication of sustainable or 'equilibrium exchange rates'. John Williamson for example has suggested that this would be an appropriate initiative for the IMF

\footnotetext{
${ }^{1}$ Portes and Rey (1998) for example.

${ }^{2}$ When asked whether a slump in currencies caused by a war in the Middle East might lead central banks to intervene in the markets, Mr Duisneberg said, "I wouldn't think so".
} 
which, as part of its new medium term strategy, aims to involve itself more closely with the affairs of G8 countries. It has been officially argued, however, that any such steps would be unattractive - even embarrassing - for the ECB and the Fed, neither of which have the exchange rate as a policy target. Both players reserve the right to intervene at values which they may judge to be inappropriate: but, save for such ad hoc intervention, things should be left to market forces.

In financial markets where portfolio management is delegated to agents with private information, traders will be subject to monitoring rules. Since trades involve signalling the quality of the trader as well as the asset, Dasgupta and Prat (2005, 2006) have shown that even traders who are being optimally monitored will fail to push prices to equilibrium; and, though they begin trading sincerely, they will eventually 'follow the herd'. With ad hoc monitoring rules, the risk of market inefficiency is much greater, as Grossman and Zhou (1993) showed for 'drawdown rules' (that involve firing traders who lose more than a given percent of previous peak value for the portfolio).

In this short paper, there are two types of trader - bulls and bears - subject to ad hoc monitoring rules, and there is excess volatility as monitoring rules lead to the 'churning' of traders. This is something of a caricature: but it allows us to show how official action can have self-fulfilling effects if market composition shifts in ways that support official stabilization. ${ }^{3}$ The action we discuss is more than merely announcing equilibrium rates, however: it involves stabilizing intervention if and when the rate moves outside what Williamson has labelled 'monitoring bands'.

The paper proceeds by first indicating how the entry and exit of bulls and bears adds to the volatility of an exchange rate driven by random walk fundamentals. In section two, in addition, it is shown how the "churning" of traders can generate excess volatility. Can this ex-ante excess volatility - induced by trading strategies and noisy fundamentals - be reduced by implementing a monitoring band? We argue that it can when the monitoring band interacts with the monitoring rules in the market; so the effect of the policy is to affect the endogenous selection of traders whose expectations match official intervention. The paper concludes that current policy may be missing a free lunch - and speculates on why reform may not be incentive compatible.

\subsection{One-Time Switching of "bear" and "bull" traders in the market}

Let exchange market traders be of two types, "bears" who are pessimistic about the trend-value of domestic currency and "bulls" who are optimistic. It is assumed that switches between employing one or the other types of trades are driven by public signals: when losses from some previous market peak hit the limit set by a "drawdown law" for example, as in Grossman and Zhou (1993). If draw-down rules are the

\footnotetext{
${ }^{3}$ See Krugman and Miller (1992) for a similar argument in connexion with target zones.
} 
same across the market then the market as a whole will switch at once, so traders at any point of time will be homogenous - which is what we assume.

Before characterizing the behavior of exchange rates when all traders are of one type or the other, we must point out that our algebraic treatment follows standard practices in measuring the exchange rate as the domestic currency price of foreign currency. So, in Europe for example, $s$ will represent the $(\log )$ price of a dollar in euros. $^{4}$

In this notation domestic currency bears will add an appreciation premium to the mathematical expectations of exchange rate change, despite fundamentals following random walk, so :

$$
E^{P} d s / d t=E d s / d t+\mu,
$$

where $E^{P}$ indicates bears' expectations, $E$ the rational expectations and $\mu$ the appreciation premium. Similarly, the change in the exchange rate expected by bulls will lie below its mathematical expectation change by a term that represents their bullishness for domestic currency, which for convenience we also assume to be $\mu:^{5}$

$$
E^{O} d s / d t=E d s / d t-\mu .
$$

If bears are permanently in the market, the arbitrage condition for the exchange rates is simply:

$$
\frac{1}{2} \sigma^{2} s_{P}^{\prime \prime}(x)+\mu=\frac{1}{\beta}\left(s_{P}(x)-x\right),
$$

where subscript $P$ represents bears. With no-bubble-condition, (2) has the solution:

$$
s_{P}(x)=x+\beta \mu .
$$

So with bears permanently in the market, exchange rates have a misalignment of $\beta \mu$. (Likewise, the exchange rates with bulls permanently in the market are given by $s_{O}(x)=x-\beta \mu$, where subscript $O$ indicates bulls).

Figure 2 illustrates how the presence of bears and bulls affects exchange rates. Using the horizontal axis to represent fundamentals and vertical the exchange rate, the 45-degree line $F F$ represents the fundamental value for exchange rates (when $\mu=0$ ). The two other parallel lines $P P$ and $O O$ indicate respectively when local currency bears or bulls are in the market. To see why $P P$ lies above the $45^{\circ}$ degree line, for example, note that - in this two currency context - a euro pessimist is a dollar optimist: so the presence euro pessimists will drive the dollar to a premium. (Conversely, for $O O$, the presence of euro optimists drives the dollar to a discount). Notice that, because there is no switching, the volatility of exchange rates is not affected in either case.

\footnotetext{
${ }^{4}$ Note, however, Figure 1 uses the opposite convention and measures the dollar price of the Euro.

${ }^{5}$ The "observational equivalence" between trend expectation and risk aversion is noted further below.
} 


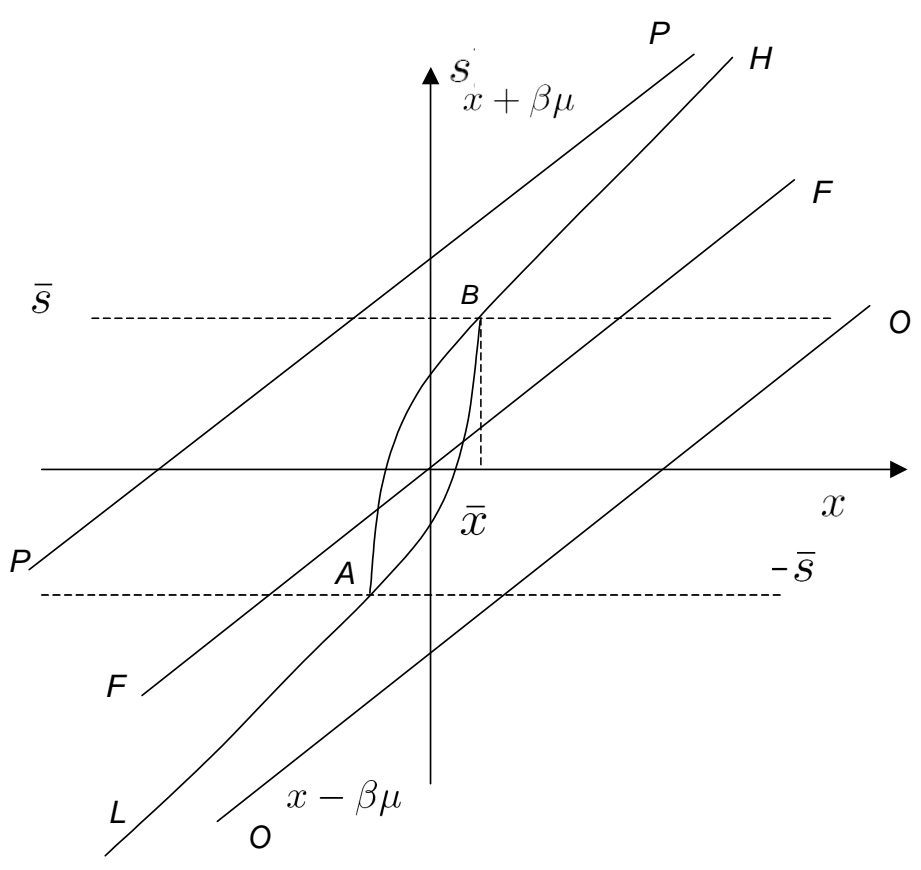

Figure 2: Exchange Rate Dynamics with One-Time Switching of Bulls and Bears 
How will switching between trader types impact on exchange rates and currency volatility? To start with, consider the case of a one-time switch. Assume that for high values of $s$ only euro-pessimists will be in the market: but they will be replaced by euro-optimists if and when the value of $s$ falls to $-\bar{s}$. Symmetrically, only eurooptimists will be in the market for low $s$, with a switch in market composition at $\bar{s}$. On these assumptions, the solutions for the exchange rates (formally derived in Appendix A) are as shown in Figure 2.

The concave curve $A B H$ represents solution under bears when they are expected to be driven out the market at $A$. The exit of bears at $A$ is locally irreversible, so only value matching applies. Since there will be no bulls in the market for exchange rates above $\bar{s}$, bears' solution approaches $P P$ asymptotically. (The exchange rate under bulls is sketched symmetrically, shown by the convex curve $B A L$.) It is clear from the figure that within the bounds of $-\bar{s}$ and $\bar{s}$, exchange rates exhibit hysteresis: if bears are in the market, they will stay there until $-\bar{s}$ is reached (vice versa for the bulls). Finally, note that this single cycle entry and exit generates significant persistence in exchange rate misalignment: take the bears' solution for example, as long as $x$ is sufficiently larger than $\bar{x}, s_{P}(x)-x \approx \beta \mu$.



Figure 3: Exchange Rate Volatility with One-Time Switch between Bull and Bears. 
Another interesting feature of exchange rates with entry and exit is that they exhibit excess volatility. The instantaneous volatility of the exchange rate is simply given by the absolute value of its slope. Since the exchange rate under bears is concave and asymptotically approaches the 45-degree line, its short run volatility is everywhere above the fundamental solution. This is shown in Figure 3, where volatility under bears starts at a higher level and declines asymptotically towards 1 . The reason why switching adds to volatility is easy to see. Starting at $L$, for example, where the market is dominated by euro-optimists, consider an increase in the variable $x$, representing fundamentals. The dollar will increase both for fundamental reasons and also because the market anticipates that the euro-optimists will lose their jobs. The same principle applies for repeated switching under a draw down rule, as is shown in the next section.

\subsection{Repeated Switching}

Exchange rate solutions when traders are hired and fired under a draw-down rule are derived formally in Appendix B. Figure 4 illustrates two solutions lying between fundamental values of $y$ and $z$, which act as triggers for firing and hiring as the vertical distance between the exchange rates $L$ and $H$ corresponds to the draw-down rule of Grossman and Zhou (1993). ${ }^{6}$ Thus the concave solution labelled $S_{P}^{R}$, shows the outcome for the exchange rate when traders are all euro-pessimists who fear losing their jobs at $L$ where fundamentals have moved against the dollar in favour of the euro. By symmetry, the solution $S_{O}^{R}$ shows how the exchange rate will behave when trades are being conducted by euro-optimists fearing to lose their jobs at $H$.

Together these segments describe a range of hysteresis rather like that in the one-time switching case shown in the previous figure. The difference here is that these linked line segments will - when fundamentals move sufficiently in the direction predicted by current traders - slide between the parallel lines labelled $S_{U I}$ and $S_{L I}$ to which they 'smooth paste' as shown in the figure. At $H$ for example a positive shock will lead to the segments sliding up along the envelope, while a negative shock involves no sliding as the rate moves down $S_{P}^{R}$.

The smooth pasting reflects the fact that traders will not lose their jobs if the rate moves in the direction that they have been forecasting; and the sliding reflects the shifting of the trigger for the drawdown rule as a new currency peak is realized. The reason that the envelope lines $S_{U I}$ and $S_{U I}$ lie inside $P P$ and $O O$ is because traders are never sure to keep their jobs. On $S_{U I}$ for instance euro pessimists are in place, but they are not permanently in place: they will expect to lose their jobs if fundamentals move fractionally against the dollar.

Since both $S_{O}^{R}$ and $S_{P}^{R}$ are steeper than $45^{\circ}$, except at the extremities where they

\footnotetext{
${ }^{6}$ Note that in the notation of Appendix B $\Delta x=z-y$.
} 


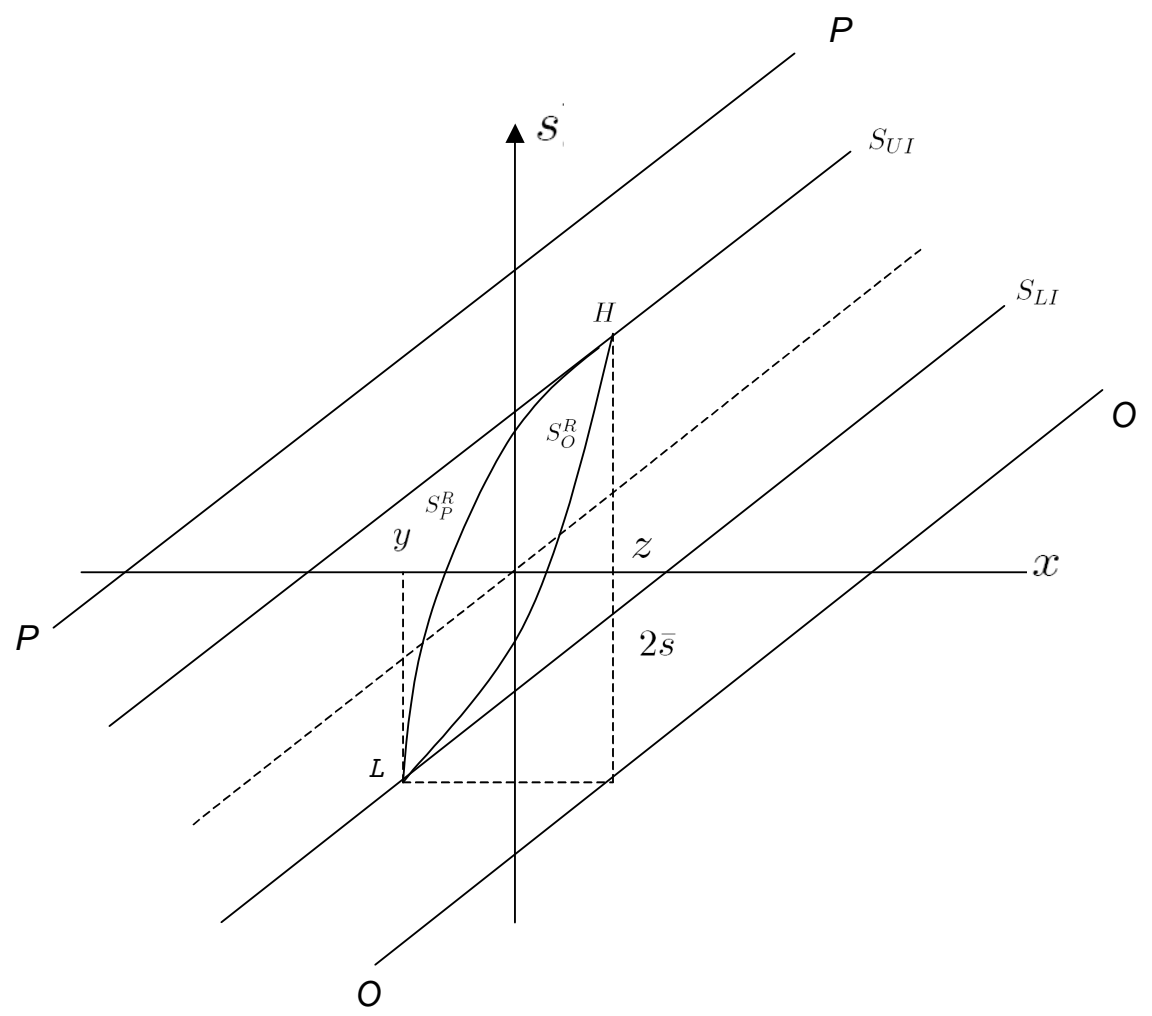

Figure 4: Repeated Switching. 
smooth paste, we conclude:

Proposition 1: For repeated switching, there is excess volatility of exchanges rates almost everywhere.

The exchange rate effects of repeated switching between bulls and bears as shown in Figure 4 closely resembles that described in Krugman and Miller (1993, Figure A2) where the switching is between risk averse wealth owners and risk neutral stop lost traders subject to draw-down rules. This is not coincidence: it reflects the fact that, in the stochastic framework we are using here, risk aversion manifest itself as a trend. This "observational equivalence" means that one may be able to apply the results we obtain here to the case where heterogeneity reflects risk preferences and not optimism of pessimism per se. We return to this point in conclusion.

\section{$2 \quad$ Effects of imposing a monitoring band}

How might the implementation of a monitoring band along the lines advocated by John Williamson (1998) affect the excess volatility described in the previous section? To answer this question we assume that:

( $i$ ) the monitoring band - within which there is no intervention - lies symmetrically around the origin with half-width $\bar{s}$;

(ii) mean-reverting intervention occurs outside these limits. For concreteness we assume that above $\bar{s}$ a negative trend of $-\mu d t$ is applied to fundamentals, and conversely a positive uptrend of $\mu d t$ below $-\bar{s}$ : but it could take the form of randomized intervention with the same expected value;

(iii) the implementation of this intervention triggers a switch in market composition at $\bar{s}$ and $-\bar{s}$ : the monitoring band is, so to say, a trigger for monitoring rules.

If the market is populated with euro-pessimists, for example, and $s$ rises to $\bar{s}$ leading to official intervention to check the rise in the dollar, this switches market composition to euro-optimists. In the terminology of Sarno and Taylor (2001; 2002, Ch. 7), we are effectively assuming that intervention policy acts as coordinating device across all agents in the market. Is this what occurred in September 2000 when an official action was taken to stop the slide in the euro?

The formal results in Appendix $\mathrm{C}$ are illustrated in Figure 5, where the schedule $A B H$ indicates the solution when traders are euro-pessimists and $B A L$ when trades are being carried out by euro-optimists. Note that outside the monitoring band there are no switches and the traders in place have beliefs that coincide with official policy. The linked solution segments $S_{P}^{M}$ and $S_{O}^{M}$ indicating the presence of hysteresis, smooth paste against the top and bottom of the band respectively (between the

fundamental triggers $\pm \bar{x}$ ). They are therefore a good deal flatter than the sliding segments in the previous section. This may be summarized as follows: 


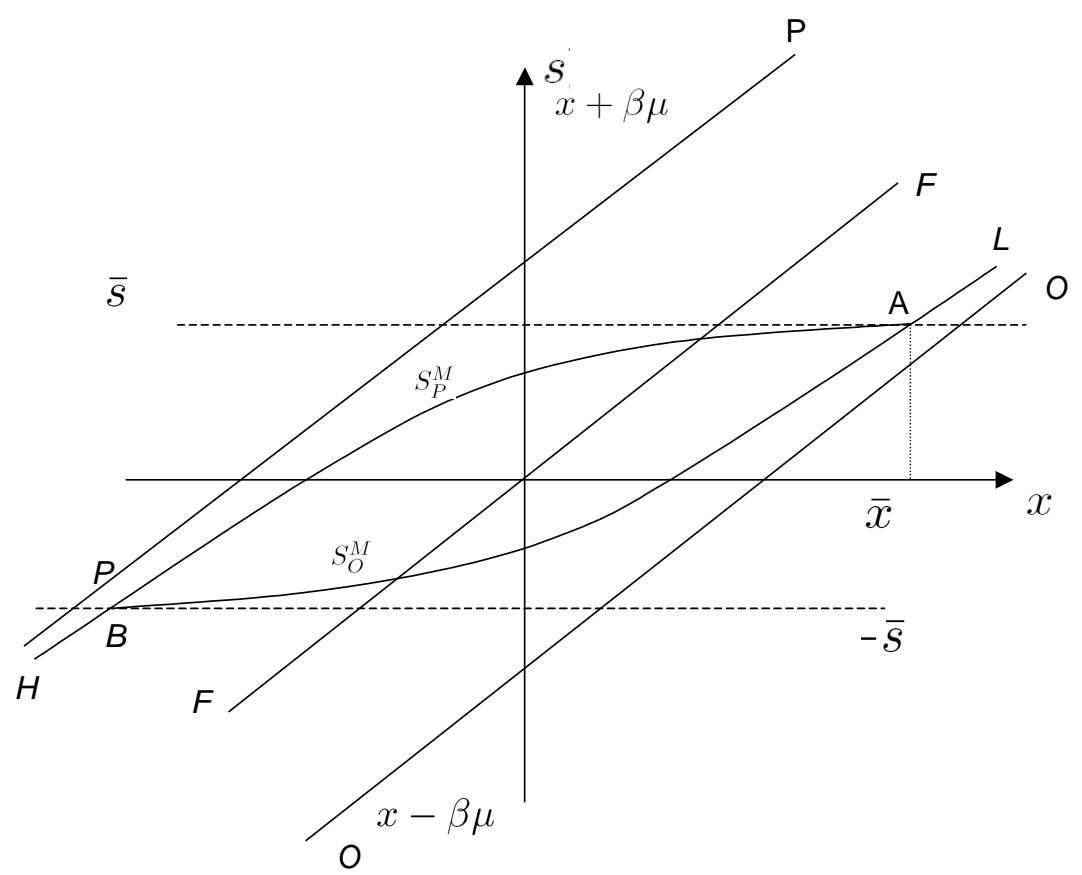

Figure 5: Entry and Exit of Bulls and Bears with a Monitoring Band

Proposition 2: With a credible monitoring band, the volatility of exchanges rates is everywhere less than one.

As $A B H$ is concave and asymptotically approaches $x+2 \beta \mu$ when $x \rightarrow-\infty$, the slope of $A B H$ is everywhere less than 1 . This implies that excess volatility for the bear's solution with monitoring band is substantially reduced, indicating the stabilizing power of the monitoring band. The same argument applies to the exchange rate solution for bulls $A B L$. These short run volatilities are shown in Figure 6 .

Our results involve a good deal of hysteresis. This would not be true if traders were to be switched in the middle of the band. This is another possibility that remains to be explored along with variations in the size of the draw-down rule. 


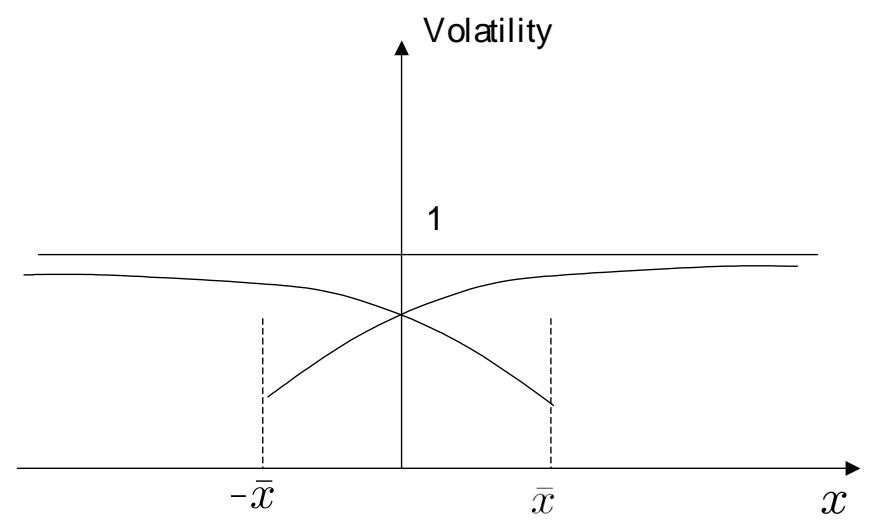

Figure 6: Exchange Rate Volatility with Entry and Exit of Bull and Bears and a Monitoring Band 


\section{Conclusions}

In September 2000, Central Banks successfully used coordinated intervention to stabilize the euro/dollar exchange rate. We have shown how, via the endogeneity of market composition, monitoring bands offer the prospect of harnessing private sector expectations in support of official policy on a more regular basis. So it seems that the laissez faire policy currently adopted by the ECB and the Fed fails to exploit a virtuous circle of self-fulfilling expectations. We are led to ask therefore whether the proposed reform is incentive compatible for the major players, including the US Fed.

Note that the model used above is based on heterogeneity beliefs about currency trends and it is virtually equivalent to the case where heterogeneity reflects risk preferences, between wealth owners and hedge funds for example. Could this interpretation provide an answer to why the free lunch still lies on the table? We have shown how churning of traders under draw-down rules generates excess volatility (and how monitoring bands can reduce this). But it is well known that the profitability of hedge funds increases with asset price volatility. So if owners of hedge funds have substantial influence on policy, it is difficult to believe that they will be enthusiastic supporters of regimes promising exchange rate tranquillity.

Further research using this alternative characterization of heterogeneity may be well be worth while. Note, however, that the zero profit assumption used in Krugman and Miller (1993) would need to be modified to allow for supernormal profits by hedge funds. 


\section{References}

[1] Dasgupta, A. And A. Prat (2006): "Financial equilibrium with career concerns". Theoretical Economics, 1, 67-93.

[2] Dasgupta, A. And A. Prat (2005): "Asset price dynamics when traders care about reputation" Mimeo. LSE.

[3] Grossman, S. J. And Z. Zhou (1993): "Optimal Investment Strategies for Controlling Drawdowns", Mathematical Finance, 3 (3), 241-276.

[4] Krugman, Paul and M. H. Miller (1993): "Why Have a Target Zone?", CarnegieRochester Conference Series on Public Policy, vol. 38, pp. 279-314.

[5] Portes, R., And H. Rey (1998): "The emergence of the euro as an international currency", Economic Policy, 26, 307-343.

[6] Sarno, L. and M. P. Taylor(2001): "Official Intervention in the Foreign Exchange Market: Is It Effective, and, If So, How Does it Work?", Journal of Economic Literature, vol 39, 839-68.

[7] Sarno, L. And M. P. Taylor (2002): The Economics of Exchange Rates. Cambridge and New York: Cambridge University Press.

[8] Williamson J. (1998): "Crawling Bands or Monitoring Bands: How to Manage Exchange Rates in a World of Capital Mobility", International Finance, 1 (1), 59-79. 


\section{Appendix}

\section{A One-Time Switching}

With bears in the market (i.e., $s(x) \geq-\bar{s}$ ), the exchange rate is a solution to (2):

$$
s_{P}(x)=x+\beta \mu+A_{+} \exp (\lambda x)+A_{-} \exp (-\lambda x),
$$

where $A_{ \pm}$are two arbitrary constants to be determined by boundary conditions. When bulls are in the market, the arbitrage condition is characterized by

$$
\frac{1}{2} \sigma^{2} s_{O}^{\prime \prime}(x)-\mu=\frac{1}{\beta}\left(s_{O}(x)-x\right), \text { for } s \leq \bar{s}
$$

which permits the following general solution

$$
s_{O}(x)=x-\beta \mu+B_{+} \exp (\lambda x)+B_{-} \exp (-\lambda x) .
$$

To determine completely the solutions for the entry and exit, we specify the following boundary conditions. Note first that, when $s>\bar{s}$, there are only bears in the market. So for $s \rightarrow \infty$, we must have the asymptotic condition

$$
\lim _{x \rightarrow \infty} s_{P}(x) \rightarrow x+\beta \mu,
$$

and likewise,

$$
\lim _{x \rightarrow \infty} s_{O}(x) \rightarrow x-\beta \mu .
$$

Applying (A.3) and (A.4) to (A.1) and (A.2) respectively yields $A_{+}=B_{-}=0$.

Assume that entries (and exits) are anticipated, and denote the fundamental trigger for the entry of bears (and exit of bulls) by $\bar{x}$, then no-arbitrage implies the following value matching condition

$$
s_{P}(\bar{x})=s_{O}(\bar{x})=\bar{s} .
$$

By symmetry,

$$
s_{O}(-\bar{x})=s_{P}(-\bar{x})=-\bar{s} .
$$

Solving (A.5) and (A.6) yields the solution for bears

$$
s_{P}(x)=x+\beta \mu-\frac{2 \beta \mu \sinh (\lambda \bar{x})}{\sinh (2 \lambda \bar{x})} \exp (-\lambda x), \quad x \geq-\bar{x},
$$

where $\sinh (\cdot)$ is a hyperbolic sine function. The solution for bulls is

$$
s_{O}(x)=x-\beta \mu+\frac{2 \beta \mu \sinh (\lambda \bar{x})}{\sinh (2 \lambda \bar{x})} \exp (\lambda x), \quad x \leq \bar{x},
$$

and the entry and exit trigger can be determined by

$$
\bar{x}+\beta \mu-\frac{2 \beta \mu \sinh (\lambda \bar{x})}{\sinh (2 \lambda \bar{x})} \exp (-\lambda \bar{x})=\bar{s} .
$$




\section{B Repeated Switching}

The solutions $S_{U I}$ and $S_{L I}$ will lie in the interior of the solution $S_{U}$ and $S_{L}$ (corresponding to $O O$ and $P P$ in Figure 4):

$$
\begin{aligned}
S_{U} & =x+\beta \mu & & S_{L}=x-\beta \mu \\
S_{U I} & =x+\beta \mu^{\prime} & & S_{L I}=x-\beta \mu^{\prime}
\end{aligned}
$$

where $0<\mu^{\prime}<\mu$ and $\mu^{\prime}$ need to be determined.

The solutions for $S_{P}^{R}$ and $S_{O}^{R}$ are:

$$
\begin{aligned}
S_{p}^{R} & =x+\beta \mu+D_{+} e^{\lambda x}+D_{-} e^{-\lambda x} \\
S_{O}^{R} & =x-\beta \mu+A_{+} e^{\lambda x}+A_{-} e^{-\lambda x}
\end{aligned}
$$

where $D_{ \pm} / A_{ \pm}$depend on the switching point $y$ but $\mu^{\prime}$ and $\Delta x=z-y$ are independent from it. Because of symmetry we only need to solve for $S_{P}^{R}$. One condition to be imposed is value matching at $L$ :

$$
S_{P}^{R}(y)=S_{L I}(y)
$$

Given that we assume reversible switching we need to impose value-matching and smooth pasting at $H$ :

$$
\begin{aligned}
& S_{P}^{R}(y+\Delta x)=S_{U I}(y+\Delta x) \\
& S_{P}^{R}(y+\Delta x)=S_{U I}^{\prime}(y+\Delta x)
\end{aligned}
$$

From (B.14), (B.15) and (B.13) :

$$
\cosh (\lambda \Delta x)=\frac{2\left(\mu+\mu^{\prime}\right)}{\left(\mu-\mu^{\prime}\right)}
$$

Since it takes $2 \bar{s}$ to switching we derive $\mu^{\prime}$ from the following switching condition:

$$
S_{U I}(y+\Delta x)-S_{L I}(y)=\Delta x+2 \beta \mu^{\prime}=2 \bar{s}=2 \bar{s}
$$

whereas $\Delta x$ is derived by replacing $\mu^{\prime}$ in (B.16) using (B.17): ${ }^{7}$

$$
\cosh (\lambda \Delta x)=2 \frac{(2 \mu \beta+2 \bar{s}-\Delta x)}{(2 \mu \beta-2 \bar{s}+\Delta x)}
$$

${ }^{7}$ Note that the RHS in is decreasing in (B.18) $\Delta x$ and the LHS increasing in $\Delta x$ thus defining a unique solution. 


\section{Monitoring Bands and Monitoring Rules}

If $-\bar{x} \leq x \leq \bar{x}$ and bears are in the market, the exchange rate takes the same form as in (A.1) and is rewritten as

$$
s_{P}^{N}(x)=x+\beta \mu+A_{+}^{N} \exp (\lambda x)+A_{-}^{N} \exp (-\lambda x) .
$$

For $x<-\bar{x}$, the arbitrage condition for bears is modified to

$$
\frac{1}{2} \sigma^{2} s_{P}^{\prime \prime}(x)+\mu s_{P}^{\prime}(x)+\mu=\frac{1}{\beta}\left(s_{P}(x)-x\right),
$$

which permits a solution of

$$
s_{P}^{I}(x)=x+2 \beta \mu+A_{+}^{I} \exp \left(\xi_{+} x\right)+A_{-}^{I} \exp \left(\xi_{-} x\right),
$$

where $\xi_{ \pm}=\left(\mu \pm \sqrt{\mu^{2}+2 \sigma^{2} / \beta}\right) / \sigma^{2}$, and subscripts $N$ and $I$ represent with and without intervention respectively.

At $-\bar{x}$, value matching and smooth pasting conditions apply

$$
\begin{aligned}
s_{P}^{N}(-\bar{x}) & =s_{P}^{I}(-\bar{x})=-\bar{s}, \\
d s_{P}^{N}(-\bar{x}) / d x & =d s_{P}^{I}(-\bar{x}) / d x .
\end{aligned}
$$

As in the previous section, similar asymptotic condition applies to $x<-\bar{x}$, i.e.,

$$
\lim _{x \rightarrow-\infty} s_{P}^{I}(x) \rightarrow x+2 \beta \mu .
$$

By symmetry, the solution for the bulls can be constructed as

$$
s_{O}^{i}(x)=-s_{P}^{i}(x), \quad i=N, I .
$$

Together with the value matching conditions (A.5) and (A.6), one can solve for the unique set of solutions for bears and bulls under the monitoring band.

Solving these boundary conditions yields a fixed point equation for the intervention point $\bar{x}$ (identical to entry and exit trigger):

$$
\frac{1}{2}\left\{(-\bar{s}+\bar{x}-\beta \mu) \cosh ^{2}(\lambda \bar{x})+\frac{\xi_{+}}{\lambda}(-\bar{s}+\bar{x}-2 \beta \mu) \sinh (\lambda \bar{x}) \cosh (\lambda \bar{x})\right\}+\beta \mu=0 .
$$

The exchange rates under bears are

$$
s_{P}^{M}(x)=\left\{\begin{array}{l}
x+\beta \mu+\frac{1}{2}\left\{(-\bar{s}+\bar{x}-\beta \mu) \cosh [\lambda(x+\bar{x})]+\xi_{+}(-\bar{s}+\bar{x}-2 \beta \mu) \sinh [\lambda(x+\bar{x})] / \lambda\right\} \\
\text { for }-\bar{x} \leq x \leq \bar{x} \\
x+2 \beta \mu+(-\bar{s}+\bar{x}-2 \beta \mu) \exp \left(\xi_{+}(x-\bar{x})\right), \text { for } x<-\bar{x} .
\end{array}\right.
$$

The exchange rate solution for bulls can be constructed using (C.24). 